\title{
XI.-On some remarkable Circumstances tending to disguise the Presence of various Acids and Bases in Chemical Analysis.
}

\author{
By JohN SPILLER,
}

ASSISTANP IN THE CHEMICAL ESTABLISHMENT OF THE WAR DEPARTMENT.

ON commencing a series of analyses of iron ores, under the direction of Mr. Abel, I had occasion to make a number of experiments in the endeavour to obtain a more perfect and rapirl method than those hitherto employed, of separating phosphoric acid from alumina.

In the course of this investigation, I was incidentally led to bring together, in the same solution, alum, citric acid, phosphate of soda, and ammonia, the citric acid being added in order to retain in solution the alumina and phosphate of alumina. On attempting to precipitate the phosphoric acid as phosphate of baryta, I was astonished to find that no precipitate was produced, and on considering that there was present in the solution, not only phosphoric acid, but also sulphuric acid from the sulphate of alumina, I was naturally led to inquire further into the cause of sulphuric acid and baryta existing together in the same solution.

Since it was evident that this extraordinary result could only be due to the presence of citric acid, a series of experiments were instituted, in order to determine the influence which citric acid exerts on the formation of precipitates in chemical analysis, the results of which I will now proceed to describe.

In order to ascertain the conditions under which the precipitation of sulphate of baryta may be prevented by citric acid and the citrates, sulphate of soda was taken as the source of sulphuric acid, and nitrate of baryta as a convenient salt of that base.

To a mixed solution of sulphate and citrate of soda a small quantity of nitrate of baryta was added; the result was, the formation of a bulky white precipitate of citrate of baryta, which immediately redissolved to a clear solution on agitation. On continuing the addition of successive portions of nitrate of baryta, a point was at last arrived at, when a permanent precipitate of sulphate of baryta made its appearance.

In the next experiment, the object was to ascertain the extent to which citrate of soda will prevent the precipitation of sulphate of baryta, and, for this purpose, weighed quantities of each of the substances were employed. 
To a known quantity of citrate of soda, sulphate of soda was gradually added, until, on testing a portion of the mixture, the sulphuric acid was immediately indicated by a drop of solution of nitrate of baryta ; and it was found that this point was attained when the equivalents of citric acid to sulphuric acid were in the relation of $1: 3$.

In order to confirm this result, equal weights of carbonate of soda were saturated, the one with sulphuric acid, the other with citric acid; on mixing these the salts in solution consisted of

$$
3\left(\mathrm{NaO}, \mathrm{SO}_{3}\right)+3 \mathrm{NaO}, \overline{\mathrm{Ci}} \text {. }
$$

In such a solution, nitrate of baryta gave no permanent precipitate, but on increasing the proportion of the sulphate of soda, the sulphuric acid was immediately rendered apparent.

With a view to determine whether citric acid exercises a similar protective action over baryta, as it does over sulphuric acid, the experiment was reversed, and the citrate of soda added to the salt of baryta. To a known quantity of citrate of soda, nitrate of baryta was added, until a permanent precipitate of citrate of baryta was formed; the solution at this stage containing

$$
2(3 \mathrm{NaO}, \overline{\mathrm{Ci}})+3 \mathrm{BaO}, \overline{\mathrm{Ci}} \text {. }
$$

On adding a little sulphate of soda, an immediate precipitate of sulphate of baryta was produced.

In connexion with this experiment, it may be stated, that the solution of the double citrate of baryta and soda deposited nearly the whole of the citrate of baryta on standing.

It would appear, therefore, that the protective action is on the side of the acid rather than that of the base.

The conditions under which the precipitate of sulphate of baryta is produced, in a solution made according to the first experiment, are the following:-by boiling, when sulphate of baryta is immediately precipitated; by the addition of an excess of nitrate of baryta ; by the addition of hydrochloric, acetic, tartaric, or oxalic acid. The sulphate of baryta, when precipitated from solution by either of these agents, is in a peculiar flocculent condition, totally dissimilar in appearance from that obtained under ordinary circumstances; so finely divided is it, that it readily passes through a filter of Swedish paper. Prolonged boiling, with or without hydrochloric acid, has little effect in changing its physical character. 
Citric acid itself has no effect in preventing the precipitation of sulphuric acid by a salt of baryta; and this is probably the reason why the addition of acids to a solution containing a neutral citrate is immediately followed by a precipitation of sulphate of baryta.

These results having been obtained by the use of a baryta salt, it became interesting to know whether the sulphates of strontia and lead were, in like manner, prevented from precipitation by citrate of soda.

To a mixture of sulphate and citrate of soda, nitrate of strontia was added, when no indication of sulphuric acid was observed, the salt of strontia behaving in a precisely similar manner to that of baryta under the same circumstances.

Nitrate of lead substituted for the baryta or strontia in these experiments gave the same result, no sulphate of lead being produced. But sulphate of lead differs from the sulphates of baryta and strontia, in being soluble, after precipitation, in citrate of soda.

My attention was next directed to the power which the citrates possess in influencing the general reactions which characterize the acids and bases in the ordinary course of chemical analysis.

Baryta.-Of the compounds of baryta there is not one that is precipitated when the acid is previously combined with citrate of soda, whether the reaction of the solution be acid or alkaline. The salts of this base, which have been made the subject of experiment, are, besides the sulphate, the phosphate, carbonate, borate, oxalate, tartrate, citrate, and fluoride. With the exception of the sulphate, all these compounds, after precipitation, are again dissolved in neutral citrate of soda.

Strontia.-The acids which were tried in connexion with this base were sulphuric, phosphoric, and carbonic, all of which compounds were retained in solution.

Lime.-Of the lime salts, the carbonate, phosphate, and oxalate were found to follow the general rule, both with respect to prevention of precipitation, and in being redissolved in citrate of soda if once produced.

The fact of oxalate and phosphate of lime being soluble in citrate of soda, suggests the possibility of this salt being employed with advantage in diseases arising from the formation of these descriptions of calculi.

As lime is the reagent generally recommended for the detection of citric acid, it is important to bear in mind that the citrate of 
lime will not be thrown down on boiling, unless the chloride of calcium be added in sufficient quantity to decompose the greater portion of the alkaline citrate, since the double citrate of lime and soda does not give any precipitate on boiling.

Magnesia.-The salts of this base appear to be less affected by the presence of citrate of soda, hydrate of magnesia and the ammonio-phosphate of magnesia being precipitated as usual. The phosphate and carbonate are, however, held in solution.

The next group, consisting of the sesquioxides of iron, chromium, and aluminium, are not precipitated by their usual group-testammonia. The phosphates of these oxides are also retained in solution by citrate of soda.

Chromium, in the state of chromic acid, is not detected by lead, silver, bismuth, or baryta. The chromate of lead does not even appear on the addition of acetic acid.

Sesquioxide of Iron.-A solution of sesquichloride of iron is not precipitated by ferrocyanide of potassium, benzoate, or succinate of ammonia; nor is the coloured reaction produced with acetate of potassa, ferricyanide, or sulphocyanide of potassium. The reaction between sesquichloride of iron and ferrocyanide of potassium is curiously modified. On mixing these two solutions in the presence of an alkaline citrate, a yellow solution is formed, which becomes deep blue in colour on largely increasing the amount of ferrocyanide of potassium; no precipitate of Prussian blue is produced until hydrochloric acid in excess is added.

From the fact that a salt of iron does not precipitate benzoic and succinic acids in presence of a citrate, the detection of these acids becomes exceedingly difficult, as we are not able to avail ourselves of any of the methods previously adopted for the decomposition of the citrate.

The group comprehending the protoxides of iron, manganese, nickel, cobalt, and zinc, are variously affected by their group-test - sulphide of ammonium. The sulphides of nickel, cobalt, and zinc are precipitated as usual; sulphide of iron incompletely; and sulphide of manganese not at all.

Protoxide of Iron.-Neither potassa nor ammonia will precipitate this base in the presence of citric acid. The alkaline product so obtained gives, with sulphide of ammonium, if dilute, a dark olive-green solution, but if more concentrated, an immediate precipitate, with a supernatant liquid of that colour. The sulphide of iron comes down, however, on boiling, or on standing for a 
sufficient length of time in the cold. The protophosphate of iron is held in solution, and the reaction between ferricyanide of potassium and a protosalt of iron is entirely masked.

Oxide of Manganese.-The most striking result observed under this head relates to the behaviour of the sulphide. The fleshcoloured precipitate usually obtained on adding sulphide of ammonium to a salt of manganese, does not appear under these circumstances, and the sulphide, after precipitation, is freely soluble in an alkaline citrate. If citrate of ammonia be the solvent employed, vapour of sulphide of ammonium is evolved on boiling. The protoxide of manganese is not precipitated by potassa, nor the carbonate by carbonate of soda, in presence of a soluble citrate. On exposure to air, the brown binoxide of manganese separates from the former only of these solutions.

Oxide of Nickel.-A salt of nickel gives, with sulphide of ammonium, the ordinary black precipitate of sulphide of nickel; but the other reagents, as potassa, carbonate of soda, and ferrocyanide of potassium, fail to give their usual indications.

Oxide of Cobalt.- - Solutions of this metal behave in a similar manner to those of nickel, with the several reagents just mentioned. The alkaline solution of protoxide of cobalt in potassa deposits the sesquioxide on exposure to air.

Oxide of Zinc.-This oxide resembles magnesia, in being precipitated by potassa in the presence of a citrate. The carbonate of zinc is retained in solution, but the sulphide and ferrocyanide are not influenced.

Sesquioxide of Uranium.-The phosphate and ferrocyauide of uranium are not precipitated in the presence of citrate of soda.

The metals of the sulphuretted hydrogen group are, with the exception of arsenic, precipitated as usual by their group-test. Several of their principal reactions are completely disguised, as will appear on describing these metals individually.

Oxide of Lead.-Besides the oxide itself, the sulphate, phosphate, carbonate, and oxalate, the bromide, iodide, cyanide, and ferrocyanide are all retained in solution on the addition of their respective precipitants. The only compound of lead, of those experimented upon, which made its appearance as under the usual circumstances, was the sulphide. The chromate has been already referred to as having its characters completely masked.

Oxide of Silver.--Inasmuch as the nitrate of silver is frequently eaployed for the detection and discrimination of several acids, 
both organic and inorganic, it was thought advisable to make a careful study of the more important compounds of this metal, and to trace their connexion with the ordinary process of analysis.

Nitrate of silver, if added in small quantity to a solution of neutral citrate of soda, gives rise to a precipitate, which, on agitation, is completely redissolved, but on continuing the addition of the nitrate of silver, a permanent precipitate is at last produced. As this final effect can only result in the destruction of the citrate of soda, and the removal of the citric acid as an insoluble silvercompound, it follows that no interfering influence need be anticipated in the ordinary course of testing for acids.

Among the compounds of silver, insoluble under ordinary circumstances, but which are held in solution through the medium of citrate of soda, are the carbonate, phosphate, oxalate, tartrate, and citrate. On the contrary, the sulphide, chloride, bromide, iodide, ferrocyanide, and sulphocyanide are precipitated as usual. The cyanide of silver is slightly soluble in this reagent, so that in its presence, very dilute hydrocyanic acid is not precipitated by nitrate of silver. Oxide of silver resembles magnesia and oxide of zinc in being thrown down by potassa under all circumstances.

Suboxide of Mercury.-By adding potassa to a salt of this base, in the presence of citric acid, the suboxide of mercury is resolved into protoxide, which remains in solution, and metallic mercury, precipitated in the form of minute grey globules. The chloride bromide, and iodide, corresponding to the suboxide, are precipitated in the usual manner.

Protoxide of Mercury.-The sulphide and iodide are not sensibly influenced in their precipitation; the latter is very sparingly soluble in citrate of soda, so that, in a highly dilute solution, the scarlet iodide does not make its appearance. The oxide and carbonate are retained in solution, and if to either of these alkaline liquids, ammonia, or one of its salts, be added, the white precipitate (chloride and amide of mercury) is immediately thrown down. The fact that oxide of mercury is not precipitated by carbonate of soda in the presence of certain salts, and that, on the addition of any compound of ammonia, an immediate precipitate is produced, is not new. A solution of protochloride of mercury, chloride of sodium, and carbonate of soda has long since been employed by Mr. E. O. Brown, as a test for ammonia; and so delicate is this test, that the slightest trace of ammonia is indicated by an opalescence throughout the liquid. 
Teroxide of Bismuth.-Neither this oxide nor the carbonate is precipitated if citric acid be present, nor is the terchloride of bismuth decomposed by water. The sulphide is, however, not appreciably affected.

Oxide of Copper.-This oxide and the carbonate are both retained in solution; the sulphide and ferrocyanide are precipitated in the usual manner.

Oxide of Cadmium.-The carbonate and oxide of this metal are also retained in solution, but not so the sulphide, which is completely precipitated.

Protoxide and Binoxide of Tin.-Both these oxides may be retained in alkaline solution on the addition of ammonia. The sulphides of tin are completely precipitated as under the ordinary circumstances.

Teroxide of Antimony.-The addition of citric acid prevents the precipitation by water of the terchloride of antimony, but does not interfere with the separation of this metal by sulphuretted hydrogen.

Antimonic Acid.-The precipitation of the pentasulphide of antimony does not appear to be so complete as in the case of the tersulphide; the separation of the last portion was attended with difficulty in some of my experiments.

Arsenious and Arsenic Acids.-The characteristic reactions of arsenic are modified in a remarkable manner. A solution of arsenious acid, to which citric acid or a neutral citrate has been added, is no longer precipitated by sulphuretted hydrogen in the cold or on boiling. The tersulphide of arsenic is also readily soluble in citric acid or a citrate. Hydrochloric acid added to either of these solutions, at once separates the whole of the arsenic.

The pentasulphide imitates the deportment of the tersulphide under the circumstances recorded above.

The arsenites of silver, copper, lime, and baryta, and the arseniates of lead, silver, and baryta, are entirely kept in solution.

From a consideration of the circumstances under which the sulphides of arsenic may be preserved in a soluble form, while the same compounds of tin and antimony are completely precipitated, I have been induced to attempt the analytical separation of these metals by such a process. My experiments have not yet been sufficiently numerous to enable me at the present time to point out the conditions under which their perfect separation may be 
accomplished, but I have every reason to believe that a quantitative method may be based upon this principle.

Teroxide of Gold.-The precipitation of the sulphide of gold takes place as under ordinary circumstances, but the reaction with protochloride of tin is modified to the extent of the production of a dingy green precipitate, instead of the brilliant purple of Cassius.

Binoxide of Platinum.-The bisulphide of platinum is precipitated on boiling, but not readily in the cold. The double chloride of platinum and ammonium has an increased tendency to assume a crystalline condition, perhaps on account of its formation being slightly retarded.

Finding citric acid to exert such remarkable solvent powers in many of the instances enumerated, I extended the inquiry to a few substances, where a parallel action might have been expected. With this intention, tartaric and racemic acids, glycerine, and the sugars were selected, and their interfering action in some few cases determined.

Tartaric acid prevents the precipitation of a salt of cobalt by potassa, and, in the form of a neutral tartrate, retains in solution sulphate of lead and the sulphide of manganese; Prussian blue is not formed in its presence; and the precipitation of sulphate of baryta is to a small extent retarded.

Racemic acid also slightly retards the precipitation of sulphate of baryta.

Glycerine has, seemingly, no influence in preventing the precipitation of Prussian blue, sulphide of manganese, or sulphate of baryta.

Under the head of the sugars, the only circumstance met with worthy of record, was the power of grape-sugar to prevent, in the cold, the formation of sulphide of manganese. Neither cane nor milk-sugar appeared to have any influence in this direction.

These examples fully confirm the fact long known, that many kinds of organic matter exert a very considerable influence on the formation of precipitates in analytical chemistry, and suggest the necessity of eliminating all these interfering circumstances before testing for the inorganic bases. It is, then, good policy to carry out the instructions of the analytical tables, when, after treatment of the unknown substance with hydrochloric acid and sulphuretted hydrogen, it is recommended to evaporate the filtrate to dryness and to clear organic matter, before proceeding to search for metals belonging to either of the subsequent groups. 
Before concluding the account of my experiments on the properties of citric acid, I cannot refrain from mentioning a difficulty early experienced in the detection of tartaric acid, when mixed with a large proportion of citric acid. So imperfect is the ordinary method of proceeding-that of employing a lime-salt to precipitate, in the cold, the tartaric acid as tartrate of lime-that I think it possible for citric acid to be adulterated with as much as 10 per cent. of tartaric acid, without fear of detection. Desiring to ascertain the purity of the sample with which I worked, my attention was naturally directed to this point. By the following process we are enabled to detect the presence of tartaric acid in cases where the old method would not be successfully employed.

To a concentrated aqueous solution of the acid under examination, acetate of potassa is added, and subsequently strong alcohol to the amount of an equal bulk; on stirring with a glass rod the crystalline bitartrate of potassa separates, time being allowed when small quantities only are suspected. By this means it is possible to detect a much smaller amount of this impurity in citric acid.

The same mode of proceeding may be turned to useful account, if the relation between these two acids be reversed. By first separating the tartaric acid as above, and afterwards evaporating the alcoholic filtrate over a water bath, the citric acid may be detected in the residue, by boiling with excess of chloride of calcium in the usual manner.

For the explanation of these curious phenomena I am not able to bring forward any hypothesis with the same degree of confidence that I have treated the facts themselves. The only possible way that has suggested itself to my mind, of accounting for the property possessed by citric acid, of retaining in solution substances which, under ordinary circumstances, are insoluble, is to suppose a neutral salt of citric acid to be endowed with the power of combining, on the one hand, with another salt, containing the acid of the compound whose formation is prevented, in such a manner as to produce a class of double salts of the general composition:-

$$
(3 \mathrm{MO}, \overline{\mathrm{Ci}})+3\left(\mathrm{MO}, \mathrm{SO}_{3}\right),
$$

in which the term $\mathrm{SO}_{3}$ is capable of being replaced by $\mathrm{CO}_{2}, \mathrm{CrO}_{3}$, $\mathrm{BO}_{3}, \overline{\mathrm{O}}$, \&c. ; or, in the case of $\mathrm{PO}_{5}$, a tribasic acid, one equivalent only will be required to take the place of three equivalents of $\mathrm{SO}_{3}$.

On the other hand it must be allowed, that an alkaline citrate 
has a tendency to combine with the citrate of another base, even though this latter compound be of itself but slightly soluble in water. The affinity existing between the two constituents of the double citrate, taken in conjunction with that exerted in the formation of the class of double-salts, just now referred to, might, it is suggested, determine in favour of the non-precipitation of the otherwise insoluble body involved.

The ascertained facts upon which this hypothesis has been principally constructed, may be shortly stated to consist in the power of one equivalent of citric acid to mask the reactions of either three equivalents of sulphuric or carbonic acid, or one equivalent of the tribasic phosphoric acid; while the existence of a class of double citrates, as those of soda with silver, lime, and baryta respectively, has been rendered highly probable in the course of this investigation.

Additional confirmation is also derived from the circumstance that the carbonates of baryta and lead dissolve in a neutral citrate with production of an alkaline liquid, the new metal remaining in solution in the form of a double citrate. And again, the alkaline product of the action of carbonate of soda on a salt of manganese, in presence of citrate of soda, does not deposit binoxide of manganese on exposure to the oxidising influence of the air, while another solution, containing caustic potassa in place of the carbonate, is constantly absorbing oxygen An analogous instance in the case of cobalt need scarcely be quoted. All these reactions are explicable, in my opinion, on the view advanced; the affinity of carbonic acid for these several bases, though weak, is strengthened by the formation of a citrocarbonate. 